\title{
En Face Optical Coherence Tomography Imaging of the Choroid in a Case with Central Serous Chorioretinopathy during the Course of Vogt-Koyanagi-Harada Disease: A Case Report
}

\author{
Yuki Komuku Chiharu Iwahashi Shinsaku Yano Chika Tanaka \\ Tomoya Nakagawa Fumi Gomi \\ Department of Ophthalmology, Sumitomo Hospital, Osaka, Japan
}

\section{Key Words}

Central serous chorioretinopathy · Choroid - En face optical coherence tomography · Steroids - Vogt-Koyanagi-Harada disease

\begin{abstract}
Vogt-Koyanagi-Harada (VKH) disease and central serous chorioretinopathy (CSC) develop serous retinal detachment; however, the treatment of each disease is totally different. Steroids treat VKH but worsen CSC; therefore, it is important to distinguish these diseases. Here, we report a case with CSC which was diagnosed by en face optical coherence tomography (OCT) imaging during the course of VKH disease. A 50-year-old man was referred with blurring of vision in his right eye. Fundus examination showed bilateral optic disc swelling and macular fluid in the right eye. OCT showed thick choroid, and en face OCT images depicted blurry choroid without clear delineation of choroidal vessels. Combined with angiography findings, this patient was diagnosed with VKH disease and treated with steroids. Promptly, fundus abnormalities resolved with the reduction of the choroidal thickness and the choroidal vessels became visible on the en face images. During the tapering of the steroid, serous macular detachment in the right eye recurred several times. Steroid treatment was effective at first; however, at the fourth appearance of submacular fluid, the patient did not respond. At that time, the choroidal vessels on the en face OCT images were clear, which significantly differed from the images at the time of recurrence of $\mathrm{VKH}$. Angiography also
\end{abstract}

KARGER 125:s $\quad \begin{aligned} & \text { Fumi Gomi, MD, PhD } \\ & \text { Department of Ophthalmology, Sumitomo Hospital } \\ & 5-3-20, \text { Nakanoshima, Kita-ku } \\ & \text { Osaka City, Osaka 530-0005 (Japan) } \\ & \text { E-Mail gomi.fumi@gmail.com }\end{aligned}$


Komuku et al.: En Face Optical Coherence Tomography Imaging of the Choroid in a Case with Central Serous Chorioretinopathy during the Course of Vogt-KoyanagiHarada Disease: A Case Report

suggested CSC-like leakage. The tapering of the steroids was effective in resolving the fluid. Secondary CSC may develop in the eye with VKH after steroid treatment. En face OCT observation of the choroid may be helpful to distinguish each condition.

(C) 2015 The Author(s)

Published by S. Karger AG, Basel

\section{Introduction}

Both Vogt-Koyanagi-Harada (VKH) disease and central serous chorioretinopathy (CSC) develop serous retinal detachment, and since recently, it has been well known that in both diseases, the thick choroid can be seen on optical coherence tomography (OCT) [1, 2]. However, the pathogenesis of each disease is totally different; VKH is an inflammatory disease affecting melanocytes [3], while CSC is a disorder of choroidal circulation [4]. Steroids treat VKH but worsen CSC [5, 6]; therefore, it is important to distinguish these diseases.

We report a case of VKH disease in which recurred serous retinal detachment was treated successfully with steroids, but the last recurrence did not respond to the steroids and angiographic findings suggested CSC. Here, we show the multimodal images of this case including en face OCT images during the course of VKH disease.

\section{Materials and Methods}

In this study, we use swept-source OCT (DRI OCT-1, Topcon, Tokyo, Japan), which allows superior visualization of the choroid thanks to a 1,050-nm wavelength light source and a scanning speed of 100,000 A scans/second. The axial and transverse resolution of the system was 8 and $20 \mu \mathrm{m}$, respectively. The OCT scan protocol was 3D scan covering an area of $12 \times 9 \mathrm{~mm}$ horizontal and vertical B scans. The software enabled us to reconstruct a series of en face images from 3D volume scan, where 992 consecutive en face images were created with a depth interval of $2.6 \mu \mathrm{m}$. When observing choroidal vessels, we referred to the en face images around the depth of the upper one third of Haller's layer, where the choroidal vessels were seen clearly at each visit.

A 50-year-old man was referred to the Sumitomo Hospital with blurring of vision in his right eye from 2 weeks prior. His best-corrected decimal visual acuity (BCVA) on presentation was 0.8 in the right eye and 1.2 in the left eye. Intraocular pressure was normal and no remarkable inflammation was observed in either the anterior or posterior chamber in both eyes.

Fundus and OCT examination showed bilateral optic disc swelling and exudative subretinal fluid (SRF) in his right eye. Choroid was significantly thick in both eyes: much thicker in the right eye than in the left eye (593 and $393 \mu \mathrm{m}$, respectively; fig. 1a, b).

En face OCT images showed blurry choroidal vessels and retinal pigment epithelium (RPE) folds associated with the disc swelling bilaterally (fig. 1c, d). Fluorescein angiography (FA) and indocyanine green angiography (ICGA) was performed using spectral-domain OCT (Spectralis, Heidelberg Engineering, Heidelberg, Germany). FA showed the leakage from the optic disc bilaterally and pooling of the dye in the subretinal space at the macula in the right eye (fig. 1e, f) and ICGA depicted patchy hyperfluorescent areas from the early phase and hypofluorescent dots in the late phase (fig. 1g, h). This patient was diagnosed with VKH disease and started to receive high-dose steroid pulse treatment. Methylprednisolone $(1,000$ $\mathrm{mg} /$ day) was intravenously administered for 3 days, followed by oral prednisolone for 16 
Komuku et al.: En Face Optical Coherence Tomography Imaging of the Choroid in a Case with Central Serous Chorioretinopathy during the Course of Vogt-KoyanagiHarada Disease: A Case Report

weeks tapering off from $30 \mathrm{mg} /$ day. Soon after the initiation of steroid treatment, the patient recognized a clearer visual field. Bilateral optic disc swelling disappeared and SRF in his right eye decreased. The choroid became thinner $(238 \mu \mathrm{m}$ in the right eye and $212 \mu \mathrm{m}$ in the left eye) and en face OCT showed the clearer visualization of choroidal vessels bilaterally. His BCVA recovered to 1.2 in both eyes.

However, 4 weeks after the cessation of steroids, bilateral disc swelling, thickened choroid ( $490 \mu \mathrm{m}$ in the right eye and $464 \mu \mathrm{m}$ in the left eye) and SRF in the right eye recurred. His BCVA was 0.3 in the right eye and 1.2 in the left eye. The en face images of the choroid were hazily obscured in both eyes. Systemic steroids were started again and 4 weeks later, bilateral disc swelling resolved completely with the reduction of the choroidal thickness and SRF. Two weeks later, however, SRF in the right eye recurred under the oral steroid medication without disc swelling. No leakage was apparent on FA and no hypofluorescent dots were seen on ICGA. The choroidal thickness was unchanged with normal appearance on en face images; however, after sub-Tenon's capsule triamcinolone acetonide (STTA) injection (20 $\mathrm{mg}$ ), SRF resolved. Four weeks later, SRF recurred again and to avoid the excessive use of steroids, bevacizumab was injected intravitreally; however, there were no remarkable changes. The additional STTA and the oral steroids were effective and complete resolution of $\mathrm{SRF}$ was obtained with improved vision of 1.0. Four weeks later, however, SRF recurred with pigment epithelial detachment (PED; fig. 2a) with a decreased BCVA of 0.4 despite the continuation of the oral steroids and the STTA, which were ineffective to resolve it. At this time, the choroid was not thick and en face OCT depicted the choroidal vessels clearly (fig. 2b). FA showed several leakages near the fovea and ICGA also depicted the leakage at the corresponding regions on FA with surrounding mixed hyper and hypofluorescence (fig. 2c, d). Then, the eye was diagnosed with prednisolone-induced CSC, and the intake of oral steroids was tapered. Soon SRF resolved and his BCVA in the right eye improved to 1.2.

One month after the stopping of oral steroids, bilateral inflammation in the anterior chamber occurred with bilateral disc swelling. OCT showed thickened choroid $(412 \mu \mathrm{m}$ in the right eye and $396 \mu \mathrm{m}$ in the left eye) with blurred choroidal vessels bilaterally, and then steroid therapy was restarted. Over 6 months under the low-dose oral steroids, there have been no recurrences of CSC in the right eye.

\section{Discussion}

This patient was initially diagnosed with VKH because of the bilateral disc swelling and characteristic hyporeflective dots on ICGA with thick choroid on OCT. His symptoms were quite responsive to the systemic steroids. During the course, the SRF in his right eye recurred several times without disc swelling, and it had been treated with steroids including STTA, but gradually became refractory. Finally, the discontinuation of steroids was effective to resolve the fluid. From the FA and ICGA, the origin of the SRF was considered to be choroidal vascular hyperpermeability such as CSC which developed secondary to the steroids.

Steroids are the first-line treatment for VKH. On the other hand, longer administration of steroids or STTA may cause the development or worsening of CSC. If steroids are continued even after the development of CSC, they may worsen CSC. Irreversible visual deterioration due to the damage of the RPE has been reported in the eyes with CSC secondary to steroids [7]. Therefore, we should know the early signs of the CSC in eyes with VKH treated with steroids. 
Komuku et al.: En Face Optical Coherence Tomography Imaging of the Choroid in a Case with Central Serous Chorioretinopathy during the Course of Vogt-KoyanagiHarada Disease: A Case Report

So far, only two cases have been reported from Japan in whom secondary CSC occurred during the treatment for VKH $[8,9]$. In each case, SRF had relapsed during steroid tapering (prednisolone 15 and $5 \mathrm{mg}$, respectively), and diagnosed with CSC using FA by the findings of dye leakages from the punctate hyperfluorescent spots seen at early phase. With the tapering of the steroids, SRF promptly disappeared. Both reports concluded that angiography was the definitive tool to diagnose secondary CSC.

In VKH, the characteristic FA leakage is multifocal in the early stage followed by pooling of dye in the subretinal space. Otherwise in CSC, univocal leakage from the level of RPE in the early stage is typical, but in eyes with CSC associated with steroids, the intense leakage from multiple regions is often seen; therefore, in addition to FA, the evaluation of multimodal imaging including ICGA is essential to differentiate both diseases.

On conventional OCT B scans, retinal detachment is easily detected, but it occurs in both VKH and CSC and sometimes it is difficult to distinguish them. The following are the differential points on OCT: VKH accompanies the folds of RPE, fluctuation of inner limiting membrane and subretinal septa, while PED is more common in CSC. The choroid is significantly thicker in VKH than that in CSC [10]. When diagnosing VKH and CSC based on choroidal thickness, attention is required because the choroid is usually thicker in active VKH than in CSC [6], but choroidal thickness changes during the course of VKH and at the cicatricial stage of VKH, the choroid becomes thin [2].

In our case, the noninvasive en face OCT scans of the choroid could suggest the different condition from active VKH as the cause of the recurrent SRF. Every time showing the active $\mathrm{VKH}$, the choroid was bilaterally thick and the choroidal vessels were blurred on en face OCT images. On the contrary, when SRF recurred without disc swelling, the choroidal thickness did not significantly increase and vessels were clearly traced, and such condition was considered to be associated with secondary CSC. Through the course of this patient, en face choroidal imaging is helpful to understand the disease condition. Since the en face image is the same two-dimensional image as a conventional fundus photograph and angiography, it is easy to compare them.

As histologically reported, the choroidal thickening in VKH is caused by the diffuse infiltration of lymphocytes, macrophages and epithelioid cells [11]. Macrophages and polymorphonuclear neutrophils infiltrate into the choroid accompanied by interstitial edema and vascular dilatation. Such cellular infiltration may cause the blurry choroid on en face images. In CSC, the choroid becomes thick due to the hyperpermeable choroidal vessels with an enlarged diameter but not blurry [12]. The en face OCT images of the choroid reflect those differences well and increase the accuracy of the diagnosis. We should put such advances in OCT technology to practical use.

\section{Conclusion}

Careful follow-up is warranted in VKH patients since secondary CSC may develop during steroid treatment. En face OCT observation of the choroid may be helpful to distinguish each condition prior to the angiography. 
Komuku et al.: En Face Optical Coherence Tomography Imaging of the Choroid in a Case with Central Serous Chorioretinopathy during the Course of Vogt-KoyanagiHarada Disease: A Case Report

\section{Acknowledgements}

The authors thank Dr. Masahiro Akiba for his technical assistance in the acquisition of en face images. This work was supported in part by a funding from Topcon Corporation, Tokyo, Japan. The funder had no role in the study design, data collection, analysis, decision to publish, or preparation of the manuscript. We also thank the optometrists of Sumitomo hospital for obtaining the clinical data.

\section{Statement of Ethics}

Written informed consent to publish was obtained from the patient for publication of this case report and any accompanying images. A copy of the written consent is available for review by the Editor of this journal.

\section{Disclosure Statement}

The authors declare that they have no competing interests.

\section{References}

1 Hamzah F, Shinojima A, Mori R, Yuzawa M: Choroidal thickness measurement by enhanced depth imaging and swept-source optical coherence tomography in central serous chorioretinopathy. BMC Ophthalmol 2014;14:145.

2 Nakai K, Gomi F, Ikuno Y, et al: Choroidal observations in Vogt-Koyanagi-Harada disease using highpenetration optical coherence tomography. Graefes Arch Clin Exp Ophthalmol 2012;250:1089-1095.

-3 Damico FM, Kiss S, Young LH: Vogt-Koyanagi-Harada disease. Semin Ophthalmol 2005;20:183-190.

4 Nicholson B, Noble J, Forooghian F, Meyerle C: Central serous chorioretinopathy: update on pathophysiology and treatment. Surv Ophthalmol 2008;58:103-126.

5 Sasamoto Y, Ohno S, Matsuda H: Studies on corticosteroid therapy in Vogt-Koyanagi-Harada disease. Ophthalmologica 1990;201:162-167.

6 Haimovici R, Koh S, Gagnon DR, Lehrfeld T, Wellik S: Risk factors for central serous chorioretinopathy: a case-control study. Ophthalmology 2004;111:244-249.

7 Wakakura M, Ishikawa S: Central serous chorioretinopathy complicating systemic corticosteroid treatment. Br J Ophthalmol 1984;68:329-331.

8 Aoyagi R, Sakai T, Okano K, et al: Angiography and optical coherence tomography findings in central serous chorioretinopathy associated with steroid therapy in a patient with Vogt-Koyanagi-Harada disease. Folia Japonica de ophthalmologica clinica 2013;6:451-456.

9 Yamauchi N, Sueno T, et al: A case of central serous chorioretinopathy during tapering of systemic corticosteroid for Harada disease. Japanese Journal of Clinical Ophathalmology 2007;61:1909-1912.

10 Lin D, Chen W, Zhang G, et al: Comparison of the optical coherence tomographic characters between acute Vogt-Koyanagi-Harada disease and acute central serous chorioretinopathy. BMC Ophthalmol 2014;14:87.

11 Inomata H, Sakamoto T: Immunohistochemical studies of Vogt-Koyanagi-Harada disease with sunset sky fundus. Curr Eye Res 1990;9:35-40.

-12 Jirarattanasopa P, Ooto S, Tsujikawa A, et al: Assessment of macular choroidal thickness by optical coherence tomography and angiographic changes in central serous chorioretinopathy. Ophthalmology 2012;119:1666-1678. 
Case Reports in

Ophthalmology
Case Rep Ophthalmol 2015;6:488-494

DOI: $10.1159 / 000443264$

(C) 2015 The Author(s). Published by S. Karger AG, Basel www.karger.com/cop

Komuku et al.: En Face Optical Coherence Tomography Imaging of the Choroid in Case with Central Serous Chorioretinopathy during the Course of Vogt-KoyanagiHarada Disease: A Case Report
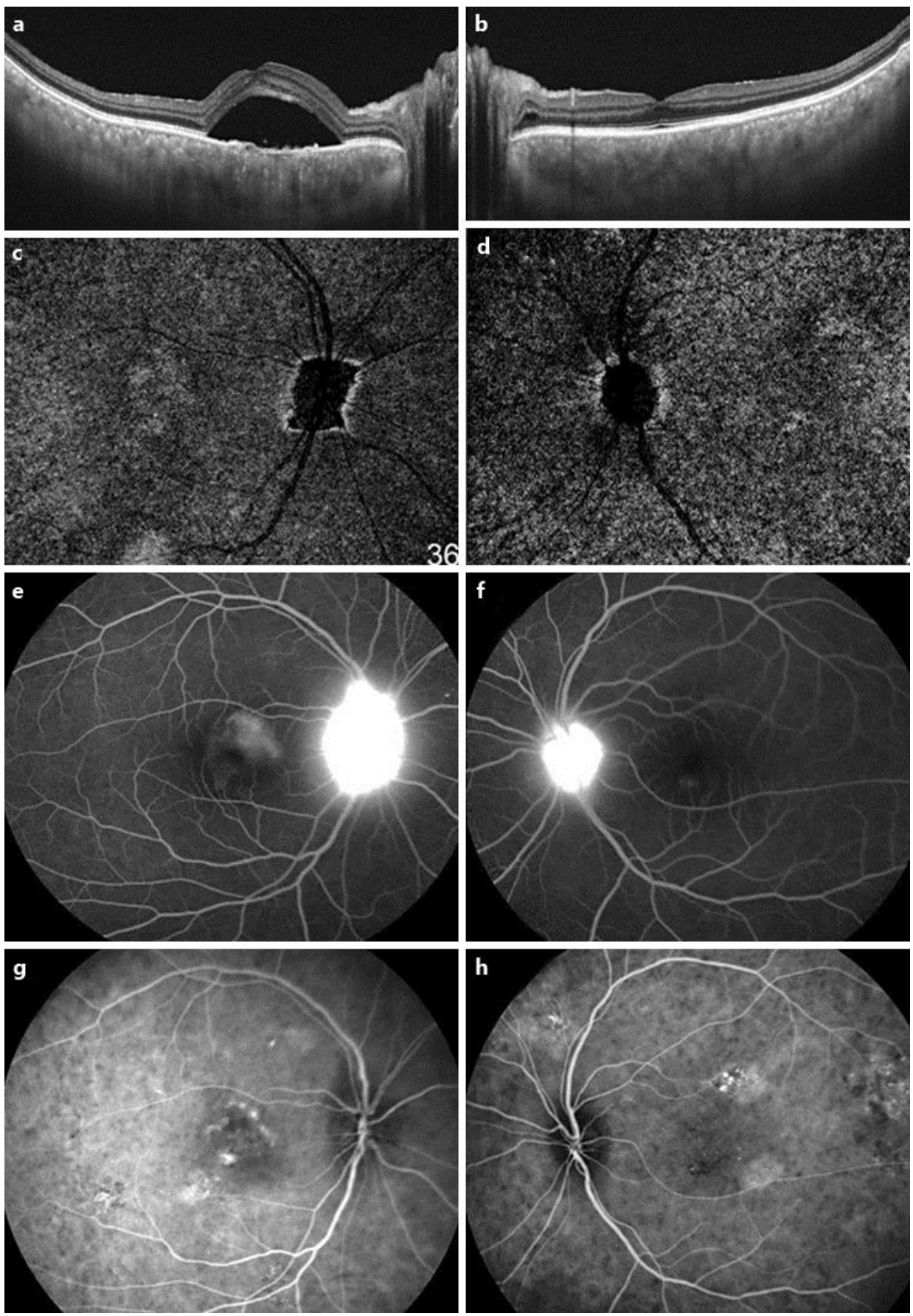

Fig. 1. Initial findings from both eyes. OCT $(\mathbf{a}, \mathbf{b})$ shows bilateral optic disc swelling and exudative SRF in the patient's right eye. The choroid is significantly thick bilaterally. En face OCT images at the level of the presumed Haller's layer of the choroid (c, d) cannot depict the choroidal vessels clearly. Optic discs are irregularly enlarged. FA shows the leakage from the optic disc bilaterally and the pooling of the dye in the subretinal space at the macula in the right eye $(\mathbf{e}, \mathbf{f})$. ICGA depicted patchy hyperfluorescent areas and hypofluorescent dots in the late phase $(\mathbf{g}, \mathbf{h})$. 


\section{Case Reports in Ophthalmology}

\begin{tabular}{l|l}
\hline Case Rep Ophthalmol 2015;6:488-494 \\
\hline DOI: 10.1159/000443264 & $\begin{array}{l}\text { ○ 2015 The Author(s). Published by S. Karger AG, Basel } \\
\text { www.karger.com/cop }\end{array}$ \\
\hline
\end{tabular}

Komuku et al.: En Face Optical Coherence Tomography Imaging of the Choroid in a Case with Central Serous Chorioretinopathy during the Course of Vogt-KoyanagiHarada Disease: A Case Report
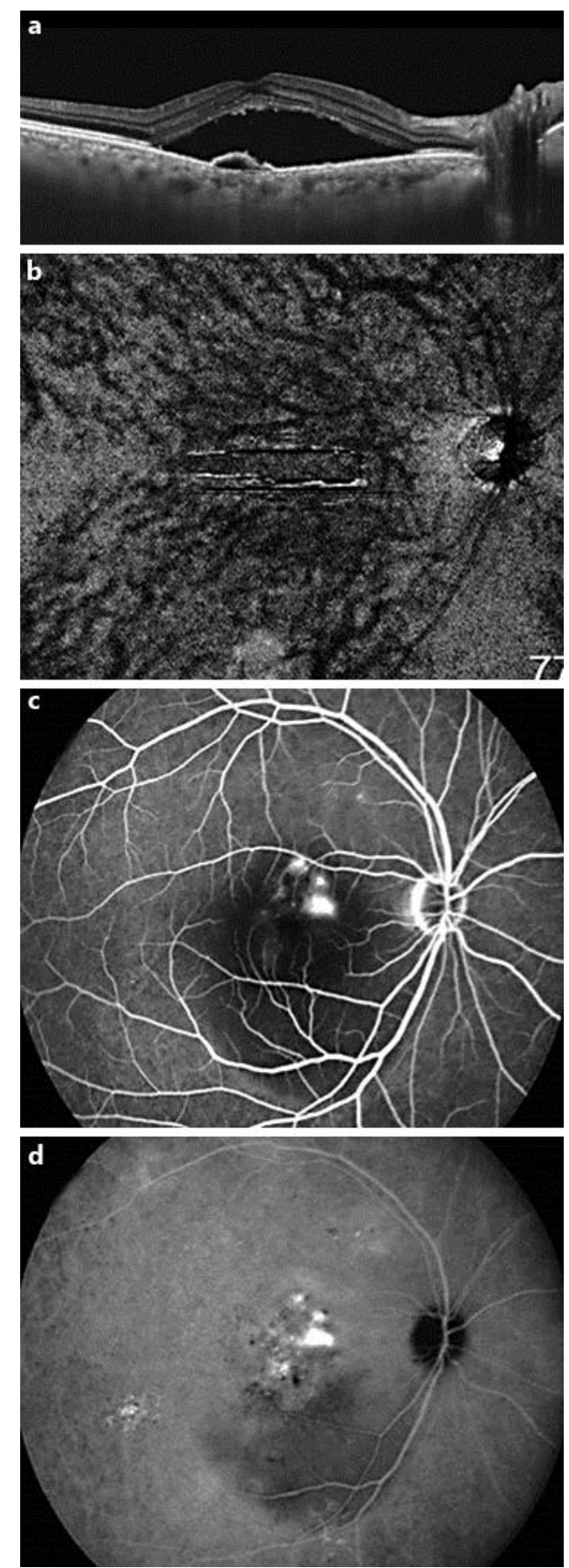

Fig. 2. At 63 weeks after initial treatment, SRF recurred with PED in the right eye, and the patient did not respond to the additional steroids (a). En face image at the level of the presumed Haller's layer of the choroid shows clearly visible choroidal vessels (b). FA (c) shows several leakages, and ICGA also depicts the leakage at the corresponding regions on FA (d). 